\title{
Protée
}

\section{Il était une fois... l'habitude}

\section{L'art de la surprise et de la répétition dans les allbums d'enfance}

\section{Nicolas Couégnas}

Volume 38, numéro 2, automne 2010

Répétition et habitude dans les pratiques quotidiennes

URI : https://id.erudit.org/iderudit/044952ar

DOI : https://doi.org/10.7202/044952ar

Aller au sommaire du numéro

\section{Éditeur(s)}

Département des arts et lettres - Université du Québec à Chicoutimi

ISSN

0300-3523 (imprimé)

1708-2307 (numérique)

Découvrir la revue

Citer cet article

Couégnas, N. (2010). Il était une fois... l'habitude : l'art de la surprise et de la répétition dans les albums d'enfance. Protée, 38(2), 59-68.

https://doi.org/10.7202/044952ar

\section{Résumé de l'article}

Cet article montre, à partir du corpus des albums d'enfance, que l'on peut distinguer plusieurs niveaux de l'habitude, plus ou moins imbriqués : une habitude inscrite dans les pratiques, un ensemble d'habitudes génériques s'exerçant au niveau discursif, et enfin des habitudes oeuvrant à fleur de texte. Dans le corpus des albums, les habitudes textuelles se manifestent par le recours à la répétition, grand pourvoyeur d'émotion et d'horizons d'attente, et la surprise, source de savoirs et de désillusion. 


\section{IL ÉTAIT UNE FOIS... L'HABITUDE L'ART DE LA SURPRISE ET DE LA RÉPÉTITION DANS LES ALBUMS D'ENFANCE}

Nicolas COUÉGNAS

\section{HABITUDES DE LECTURE}

Il y a une habitude, saine habitude, qui voit le retour chaque soir dans nombre de familles comprenant au moins un jeune enfant, d'un moment hautement ritualisé qui participe activement au cérémonial du coucher: celui de l'histoire, que l'un des parents doit raconter à l'enfant avant que celui-ci ne s'endorme. Ces histoires à lire et à dire sont aujourd'hui le plus souvent racontées avec le support d'un texte que l'on peut qualifier génériquement par le terme d'album d'enfance. L'invention ou la réinvention à partir d'un patrimoine de contes demeurent évidemment tout à fait possibles, mais l'album et ses proches cousins l'abécédaire, l'imagier, etc. paraissent offrir une créativité et une adéquation aux besoins de l'enfant difficiles à concurrencer. Or ces albums, qui rencontrent un succès éditorial qui ne se dément pas, sont eux-mêmes, en quelque sorte, un véritable temple de l'habitude. L'album en effet, à l'instar très certainement de nombreux moments de notre vie sociale, entrecroise au moins trois variétés de l'habitude possédant leur propre logique interne: i) une habitude pratique, ii) une habitude discursive et enfin iii) une habitude structurelle. Ces trois domaines déclinent les différents niveaux de pertinence du monde sémiotique emboîtés les uns dans les autres: la structure textuelle s'incarne dans des discours particuliers qui euxmêmes ne prennent sens qu'au sein de pratiques sociales spécifiques.

L'habitude comme pratique est ici celle du moment de lecture lui-même. Pour tous les parents désireux de respecter les commandements impérieux du code de bonne conduite parentale, cette habitude s'avère aussi incontournable que celle du «doudou». La lecture d'album n'est d'ailleurs pas si éloignée de l'«objet transitionnel» winnicottien (Winnicott, 1975) puisqu'il s'agit de ménager, par le recours à l'album, un espace de médiation, de transition, entre l'enfant et le monde. Si l'on exclut l'acte de lecture "libre» pratiqué par le jeune enfant non lecteur, la situation de lecture la plus courante des albums possède des caractéristiques relativement inédites, ce qui implique que l'analyse des albums ne peut se passer d'une prise en compte de la pratique interprétative dans laquelle les albums prennent sens. En premier lieu, les albums s'adressent à un public extrêmement spécifique, les enfants, et doivent donc nécessairement tenir compte de leurs compétences interprétatives et cognitives, qui ne sont évidemment pas celles des adultes. Au-delà du simple constat, on peut d'ores 
et déjà pointer le fait qu'il y a un lien direct entre les compétences enfantines et le rôle central joué par l'habitude sur les plans discursif et structurel des albums. Second point, pour les albums d'enfance proposés aux tout jeunes enfants, qui soit ne savent pas encore lire, soit sont encore très loin d'être des lecteurs experts autonomes, la situation est encore un peu plus complexe puisqu'elle implique l'association de deux acteurs dans l'acte de lecture. Il y a un lecteur adulte, qui décode le message verbal et iconique et lit l'histoire, ou fait une histoire de l'album (parfois en l'absence de texte), et un observateur enfant qui voit l'image, en décode des éléments, reçoit le récit fait par l'adulte et interagit avec lui.

Cette pratique étant une pratique de lecture, elle mobilise logiquement le second niveau des habitudes, celui des habitudes discursives. Sous cette appellation générale se classent les contraintes de discours et de genre. Or, dans les discours du type «littérature d'enfance et de jeunesse», le genre des albums, bien que l'on ait parfois du mal à le définir précisément, apparaît comme extrêmement contraint. Sans que la créativité des albums en pâtisse, ceuxci semblent obéir à un haut degré de généricité. À partir d'un corpus relativement vaste, on a pu établir un arbre générique des albums se déployant d'un socle générique, présent dans tous les volumes, jusqu'aux trois grandes ramifications possibles. En bref, on peut dire que le socle générique des albums réside en premier lieu dans la révolution médiatique qui a consisté, au début du XXe siècle, à accorder à l'image un rôle prépondérant dans certaines des productions destinées aux enfants. De fait, depuis Macao et Cosmage (1919), un album c'est avant tout une succession d'images, associées à des commentaires textuels plus ou moins discrets, qui peuvent, le cas échéant, disparaître tout bonnement, signe de leur rôle secondaire par rapport à l'image. Cette composante médiatique, en phase avec la situation de lecture et avec les compétences cognitives de l'enfant, décide en second lieu de l'organisation tactique, c'està-dire de la disposition spatiale du plan de l'expression et du plan du contenu. La tactique de l'expression des albums est aisément reconnaissable: ce type textuel est toujours constitué d'un nombre très réduit d'images, allant de l'image en double page à un maximum de deux images par page. De cette disposition du plan de l'expression découle une disposition également parfaitement linéaire du plan du contenu: la progression thématique ou narrative d'un album se fait toujours selon une progression bien réglée, chaque page apportant son lot minimal d'informations. À partir de ce socle, trois grandes ramifications sont envisageables, qui correspondent aux trois modèles auxquels les différents textes empruntent leur principe de base et leur ressort narratif fondamental, à savoir: le modèle du conte, le modèle de la boule de neige et le modèle de la surprise (Couégnas, 2010).

Enfin, au terme de ce rapide parcours, s'impose une autre forme d'habitude à l'œuvre dans la structure narrative et plus largement textuelle des albums. C'est à ce niveau que l'habitude paraît comme réellement, ou explicitement, problématisée. Dans les deux premiers cas, l'habitude se donne dans son appareil le plus simple, comme le retour du même avec un degré plus ou moins fort de variation. Pour la pratique, le même c'est la situation de lecture, les acteurs de la situation et le genre de texte exploité. La variable réside dans le choix des albums, sachant d'ailleurs que, d'une part, le fonds d'album des familles n'est pas illimité, mais que, surtout, les enfants sont de grands amateurs de lecture intensive. Ils voient avec grand plaisir se rejouer plusieurs soirs le même théâtre entre les pages des albums, capable d'engendrer la même émotion et en tout cas la même envie de lecture. Dans le genre, l'habitude s'exprime par l'identité très forte entre albums malgré la très grande productivité de l'édition. Les variations ont lieu quand la pression générique se relâche, c'est-àdire dans le choix de tel ou tel modèle, puis dans la capacité des auteurs à produire une ouvre singulière à partir des éléments génériques. Au niveau textuel, l'affaire est apparemment beaucoup plus complexe car l'habitude constitue un enjeu primordial de l'interprétation et du plaisir de lire des enfants. L'habitude devient alors un art de la répétition et de la surprise, qui sont deux ressorts efficaces de la création et de la dynamique textuelle. On doit donc envisager dans ce cas les formes de la répétition et de l'habitude dans la matière singulière de la textualité. 
Le cadre théorique et méthodologique de la sémantique textuelle développée par François Rastier offre des outils relativement précis pour appréhender ces différents mécanismes de l'habitude. Rappelons brièvement que Rastier distingue plusieurs composantes textuelles, qui ne se déploient pas générativement, comme dans le modèle de la sémiotique narrative greimassienne, mais interagissent selon des modalités en partie fixées par les normes génériques mobilisées par le texte. Ces composantes sont les suivantes:

1. La thématique rend compte des contenus investis, c'est-àdire du secteur de l'univers sémantique mis en œuvre dans le texte. Elle en décrit les unités. Par analogie, et bien qu'elle ne décrive pas spécifiquement le lexique, on peut dire qu'elle traite $d u$ "vocabulaire» textuel, dont nous détaillerons plus loin les unités (molécules sémiques, faisceaux d'isotopies, etc.). 2. La dialectique rend compte des intervalles temporels dans le temps représenté, de la succession des états entre ces intervalles, et du déroulement aspectuel des processus dans ces intervalles.

3. La dialogique rend compte des modalités, notamment énonciatives et évaluatives, ainsi que des espaces modaux $q u$ 'elles décrivent. Dans cette mesure, elle traite de l'énonciation représentée (l'énonciation réelle ne relevant pas de la linguistique, mais de la psycholinguistique).

4. La tactique rend compte de la disposition séquentielle du signifié, et de l'ordre linéaire (ou non) selon lequel les unités sémantiques à tous les paliers sont produites et interprétées.

(Rastier, 2006: 21)

La composante médiatique enfin, ajoutée plus récemment, décrit spécifiquement le plan de l'expression, les liens entre supports et types de signes exploités. On remarquera d'emblée que la surprise relève prioritairement de la composante dialogique, qui décrit les jeux énonciatifs possibles entre mondes factuels et mondes contrefactuels, alors que la répétition mobilise massivement, dans le cas des albums, la composante dialectique. La répétition est bien évidemment, par définition, également à l'œuvre au niveau thématique: les isotopies sont avant tout un phénomène de récurrence de sèmes, de molécules sémiques ou de faisceau sémantique. Néanmoins, seule la composante dialectique, qui permet de décrire la syntagmatique du texte, paraît à même de révéler l'essence d'une répétition qui ne soit pas réduite à un effet de cohérence, mais se présente comme une dynamique «dialectique» où s'entrelacent le même et le différent.

La première ambition de ce travail est d'ordre technique et méthodologique. Il s'agit de montrer que les outils descriptifs, exploités pour rendre compte des composantes sémantiques, dialectiques et dialogiques, s'avèrent pertinents pour aborder en détail les mécanismes de l'habitude, de la surprise et de la répétition. Deuxième objectif, s'attaquer au contenu de ces mécanismes, par le biais de l'analyse des deux modèles textuels les plus caractéristiques des albums d'enfance: la boule de neige et la surprise, où l'on verra que la répétition reçoit une orientation prospective et compose d'abord avec la dimension sensible, alors que la surprise est rétrospective et d'essence cognitive.

\section{ÉMOTION ET STRUCTURE DE LA RÉPÉTITION}

L'album est d'abord un art de la répétition: celleci est présente partout, derrière chaque couverture d'album, au détour de chaque tourne, page après page. La répétition n'est pas, pour autant, propre à ce type textuel. Elle est un motif narratif standard des contes, décliné à l'envi par la triplication des épreuves. Mais, dans le cas des albums, la répétition fait doublement loi: d'un côté, versant lecteur, parce que la répétition est bien, classiquement, un principe narratif puissant, générateur d'attentes, de surprises; de l'autre, versant créateur, parce que la répétition fait écho à la forme de ces ouvrages particuliers. Dit autrement, la répétition, principe organisateur du "modèle de la boule de neige», est l'un des aboutissements logiques du socle générique précédemment décrit. À chaque tourne, une nouvelle image apparaît, qui, à la fois, doit être interprétable par l'enfant comme un ensemble autonome qu'il a le temps de parcourir et à la fois prend sens par rapport à l'image précédente et à l'image à venir. De cette contrainte naît le privilège accordé à la répétition, rassurante et ludique, cellule de base pour engendrer un art de la variation susceptible de satisfaire l'enfant lecteur. La boule de neige du contenu, qui colle sans 
reste à la linéarité discrète du plan de l'expression, va de page en page, répétant son mouvement et grossissant la plupart du temps. Mais l'accroissement n'est pas la seule progression possible, le modèle connaît d'autres possibilités. À partir du modèle général, fondé par la dialectique répétition/variation, plusieurs voies sont empruntées par les albums, en fonction de ce qui varie et du type de variation. Sans que cela soit exhaustif, nous distinguons pour le moment deux possibilités. La déclinaison représente le cas le plus simple, où d'une page à l'autre un seul élément iconique diffère, entraînant une variation dialectique minimale. La même cellule narrative est reconduite inlassablement, qui laisse une variable libre à occuper. On peut citer pour exemple C'est moi le plus fort (Ramos, 2001), où un loup en mal de reconnaissance demande confirmation à chaque animal rencontré de son incontestable supériorité. Le destinataire de la question change à chaque double page, jusqu'au dénouement. Autre cas célèbre de répétition-déclinaison, celui De la petite taupe qui voulait savoir qui lui avait fait sur la tête (Holzwarth et Erlbruch, 1989). À chaque double page, la taupe demande à une nouvelle créature qui a bien pu lui faire sur la tête au début de l'ouvrage. Dans cette enquête scatologique, la variation porte à la fois sur les animaux interrogés et sur l'excrément produit par ceux-ci. On remarquera que, dans tous les cas, la variation peut être décrite comme une variation au sein d'un paradigme sémantiquement homogène, autrement dit une classe sémantique. Seul importe d'ailleurs, dans ce cas, le sème générique qui indexe chacun des animaux, ou des excréments, dans leur classe sémantique commune. Il en va autrement pour la répétition avec intensification, dont le principe justifie le terme de boule de neige. Ce second cas constitue sans conteste le sous-modèle le plus exploité par les albums d'enfance, et l'on doit d'ailleurs se demander ce qui justifie un telle popularité. Avec l'intensification, l'une des dimensions événementielles (acteurs, actions) connaît une intensité croissante, ou éventuellement une intensité décroissante, ce qui n'est qu'un point de vue différent sur un mécanisme identique. Il s'agit donc non plus simplement de parcourir le paradigme d'une classe sémantique, mais d'exploiter les sèmes spécifiques graduels qui organisent la classe sémantique et permettent de distinguer relativement, différentiellement, les éléments qu'elle contient. Les exemples ne manquent pas pour illustrer ce cas. L'intensification joue par exemple un rôle central dans La Piscine (Poussier, 2008). Dans cet album, une souris invite un lapin rose à accomplir un plongeon dans la piscine. Le lapin refuse en prétextant la peur. La souris décide de lui montrer comment faire. Elle se dirige vers le plongeoir, bientôt suivie par un gros chat gris, puis par une énorme poule rouge, puis par un renard, puis par un ours, puis par un très gros mouton, jusqu'à l'éléphant qui rejoint par la voie des airs l'ensemble des autres animaux en train de barboter dans l'eau. La piscine est pleine, le lapin se plaint alors qu'il ne peut plus plonger, alors qu'il s'y apprêtait, et sauve ainsi sa dignité de lapin. La classe sémantique centrale des animaux capables de plonger dans la piscine est organisée par un gradient, qui classe les animaux du plus petit au plus grand. La tactique du contenu, qui décide de l'ordre d'apparition des animaux, suit parfaitement l'organisation graduelle de la classe sémantique, parcourue du plus petit au plus grand. De cette première course à l'intensification découlent deux conséquences du même ordre: le nombre des animaux présents dans la piscine augmente et la place pour plonger diminue d'autant, justifiant le renoncement du lapin.

À ce stade, les graphes thématisés, utilisés par Rastier pour décrire notamment la composante dialectique, permettent d'appréhender en détail le jeu des variations et des constantes manipulées par la rhétorique de la répétition. Ces graphes sémantiques peuvent représenter une structure sémantique quelconque, c'est-à-dire des sèmes, ou des sémèmes, ou des molécules sémiques et les relations casuelles qui les unissent. Les sèmes (ou sémèmes, ou molécules sémiques) constituent les nœuds du graphe sémantique (représentés dans des cartouches rectangulaires ou entre crochets) et les relations casuelles, ses liens (représentés dans des cercles elliptiques ou entre parenthèses). Par exemple, pour représenter les aventures de T'choupi au supermarché, on notera ainsi les relations entre acteurs: 


\section{$[$ T'CHOUPI $] \leftarrow($ ERG $) \leftarrow$ PRENDRE $] \rightarrow($ ACC $) \rightarrow$ \\ [DES BONBONS] $\rightarrow$ (LOC S) $\rightarrow$ [AU SUPERMARCHÉ]}

où ERG désigne le cas ergatif (Acteur/actant agent d'un procès/processus); ACC, l'accusatif (Actant/ acteur patient d'un procès, entité qui est affectée par l'action); et LOC S, le locatif spatial.

Les exploits du lapin rose d'Audrey Poussier, dans La Piscine, seront notés ainsi:

1) [Le LAPIN $] \leftarrow[$ ATt $] \leftarrow$ [PEUR DE PLONGER DANS LA PISCINE]

2a) $[$ ANimal A] $\leftarrow$ (ERG) $\leftarrow$ P PlONGE] $\rightarrow$ (LOC) $\rightarrow$ [DANS LA PISCINE DE SURFACE S]

2b) [ANimal B] $\leftarrow$ (ERG) $\leftarrow$ [PLONGE] $\rightarrow$ (LOC) $\rightarrow$ [DANS LA PISCINE DE SURFACE S MOINS LA PLACE PRISE PAR A]

2c) [ANimal C] $\leftarrow$ (ERG) $\leftarrow$ [PLONGE] $\rightarrow$ (LOC) $\rightarrow$ [DANS LA PISCINE DE SURFACE $S-\mathrm{A}+\mathrm{B}$ ]

ETC.

3) [LE LAPIN $] \leftarrow($ NOM $) \leftarrow[$ REGARDE $] \rightarrow($ ACC $) \rightarrow[2 \mathrm{~A}, 2 \mathrm{~B}$, 2C, etc.]

4) [Le Lapin $] \leftarrow$ (ATT) $\leftarrow$ N'A PAS PeUR DE Plonger DANS LA PISCINE]

5) $\quad$ [LE LAPIN $] \leftarrow$ (ERG) $\leftarrow$ [QUITTE LE LIEU] $\rightarrow($ LOC S $) \rightarrow[$ LA PISCINE PLEINE]

où ATT désigne l'attributif (de qualité, de possession, de dépendance - relation entre une qualité attribuée et son unité récipiendaire, unité possédée/unité possédante, unité dépendante/unité dominante); et NOM, le nominatif (qui exprime un état neutre entre l'ergatif et l'accusatif).

La variation, répétée de page en page, porte sur deux des acteurs impliqués dans le graphe thématisé du plongeon. Tout d'abord, la classe sémantique des acteurs accomplissant l'acte de plonger: //souris $<$ chat $<$ poule $<$ renard < ours, mouton < éléphant $/ /$. Dans cette classe, tous les membres possèdent le sème générique «animal», le sème ergatif «plonger dans la piscine» et ils sont organisés du plus petit au plus grand par le sème spécifique graduel de la taille. Seconde classe, celle de l'acteur correspondant au locatif spatial «la piscine»: // piscine vide de surface $\mathrm{S}<$ piscine de surface $\mathrm{S}-\mathrm{A}<$ piscine de surface $(S-A)-B<$ (etc.) $<$ piscine pleine $/ /$. Le sème générique est bien sûr celui de la piscine et le sème spécifique, celui de la taille décroissante en raison du nombre de baigneurs. L'ensemble des variations apportées par la tactique dialectique linéaire aboutit à une transformation dialectique non linéaire: la transformation de l'acteur Lapin, qui perd sa molécule sémique spécifique /avoir peur de plonger/, au profit de /ne pas pouvoir plonger/ et /être rusé qui ne perd pas la face/.

Deuxième exemple, pour bien saisir les avantages de ce système de notation des actions sur un cas plus complexe et extrêmement créatif d'album. Dans Cependant... Le livre le plus court du monde (2002), le graphiste Paul Cox interroge l'essence répétitive de l'album avec talent. Au premier abord, l'album ressemble fort à une énigme. On cherche, sans les trouver, une page de couverture et une quatrième de couverture: aucune page ne se distingue des autres par les mentions habituelles, d'auteur, de titre, permettant d'identifier la couverture ou la quatrième, ou par une épaisseur particulière, toutes les pages étant également cartonnées. Il n'y a dans cet album ni début ni fin mais au contraire une succession sans fin d'images stylisées, représentant diverses activités humaines sans lien. Les images sont belles, le commentaire pour l'enfant possible, mais le sémioticien en mal d'isotopies et de cohérence textuelle reste sur sa faim. Le statut avancé par le soustitre, «livre le plus court du monde», et la mention "cependant", en haut de chaque illustration, invitent à plus de réflexion et promettent une autre subtilité de fonctionnement. Le livre le plus court du monde arrête le temps et propose au lecteur un tour du monde, dans le temps suspendu du "cependant», qui signifie en fait «au même moment, un peu plus loin autour du globe». Chaque image représentant ainsi ce qui se passe autour de la terre, au même moment, sans plus de cohérence sémantique. S'ajoute à cela l'amorce d'une autre isotopie, qui convoque le modèle de la boule de neige: on s'aperçoit que, d'une image à l'autre, la lumière croît doucement, puis décroît pendant plusieurs pages, puis augmente de nouveau, inlassablement, l'album ne présentant ni début ni fin. Les variations de la lumière d'une image à l'autre correspondent donc, conformément à ce 
que suggère le titre, aux variations de la lumière tout autour du globe, parcouru dans le temps suspendu du «cependant». Deux graphes thématisés sont nécessaires pour représenter la structure dialectique de l'album, ce qui s'y répète et s'y transforme.

$$
\begin{aligned}
& \text { GRAPHE A) } \\
& \begin{aligned}
(\text { LOC T) } & \rightarrow[\text { AUU MOMENT T }] \\
\uparrow & \\
{[\text { A }] \leftarrow \text { (ERG }) \leftarrow[\text { FAIT }] \rightarrow(\text { ACC }) } & \rightarrow \text { [QUELLUE CHOSE] } \\
\downarrow & \\
(\text { LOC S }) & \rightarrow[\text { DANS UN LIEU LN] }
\end{aligned}
\end{aligned}
$$

GRAPHE B)

$$
\begin{aligned}
& (\text { LOC T) } \rightarrow[\text { AU MOMENT T] } \\
& \uparrow \\
& {[\mathrm{B}] \leftarrow(\mathrm{ERG}) \leftarrow[\mathrm{FAIT}] \rightarrow(\mathrm{ACC}) \rightarrow[\text { QUELQUE CHOSE }]} \\
& \downarrow \\
& \text { (LOC S) } \rightarrow \text { [DANS UN ENDROIT LN }+1 \\
& \text { (une distance suffisante } \\
& \text { pour que la lumière varie)] } \\
& \downarrow \\
& \text { (RES) } \rightarrow \text { [LA LUMIĖRE VARIE] }
\end{aligned}
$$

Dans cette composition dialectique, le rôle ergatif est constant, il y a toujours quelqu'un qui fait quelque chose, mais il y a variation de l'acteur en position ergative. Chaque nouvel acteur relève de la classe des habitants situés sur un arc de cercle faisant le tour du globe: //habitant de Ln, de Ln + 1, de Ln + 2, etc //. Le rôle locatif spatial est également toujours présent, mais l'acteur est également soumis à variations // Ln, $\operatorname{Ln}+1$, de $\operatorname{Ln}+2$, etc.//. Le rôle résultatif est présent mais varie graduellement, en intensité lumineuse. En revanche, l'acteur locatif temporel est constant; il correspond à $\mathrm{T}_{0}$, le moment de l'énonciation. Il faut cette notation complexe permise par les graphes thématisés pour distinguer, sans difficulté, la variation ou le maintien des rôles narratifs, ou dialectiques, nécessaires à l'effet de répétition, des variations et permanence des acteurs.

Attardons-nous à présent sur l'essence passionnelle de la répétition et sur son omniprésence dans les albums pour enfants. Comme l'indique bien le double graphe indispensable à la représentation de l'album de
Paul Cox, le modèle de la boule de neige n'a de sens que dans une dynamique qui va de l'avant, et porte avec empressement le lecteur vers la page suivante. En d'autres termes, la répétition participe avec force à la constitution de l'horizon d'attente des albums. Les modèles de la sémiotique tensive développés par Fontanille et Zilberberg (1998) sont particulièrement appropriés pour rendre compte des tensions associées aux horizons d'attente. Mieux, on peut y trouver l'explication de l'orientation prospective de la répétition. La répétition avec intensification mobilise deux schémas tensifs qui œuvrent de conserve pour créer une amplification, c'est-à-dire une augmentation corrélée de la valence d'intensité et de la valence d'étendue (Couégnas, 2009). Le premier schéma caractérise la répétition elle-même et sa capacité à engendrer une attente croissante de la part de l'enfant: à chaque page tournée, à chaque nouvelle répétition de la cellule narrative précédemment identifiée, le phénomène s'amplifie, la surprise de la répétition monte d'un ton. Nous sommes dans le registre du «il l'a encore fait», chaque fois plus improbable, mais toujours attendu, et chaque fois plus sensationnel. Faire entrer en scène un éléphant plongeur, après que l'oursonne a plongé, est attendu par le fait même des répétitions antérieures: la répétition est en quelque sorte programmée, l'attente renforcée, mais l'arrivée de l'éléphant fait néanmoins office de coup de théâtre, chaque nouvelle surenchère apportant une intensité supplémentaire. Plus la structure se répète, plus elle est attendue, moins elle a de probabilité de se répéter: c'est chaque fois comme un coup de force de l'auteur, que l'enfant applaudit et goûte à sa juste valeur. On peut représenter la corrélation dans le schéma tensif suivant, où l'intensité correspond à la force de l'émotion suscitée par chaque nouvelle apparition et reconnaissance de la répétition, et où l'étendue correspond, d'une part, à l'augmentation de la répétition, à chaque nouvelle page, et, d'autre part, à la baisse correspondante de probabilité d'une nouvelle répétition (voir à la page suivante le Schéma de l'horizon d'attente 1).

Sur l'axe de l'étendue, les graduations de la probabilité $\mathrm{du}+$ vers le -, et du nombre de répétition $\mathrm{du}-$ vers le + traduisent le fait que la probabilité est 


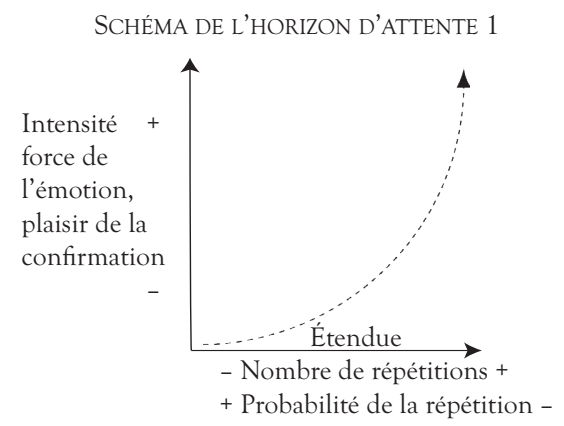

logiquement inversement proportionnel au nombre d'occurrences de la répétition.

Dans le cas de la répétition avec intensification, lorsque la boule de neige accomplit réellement son destin de boule de neige, l'amplification inhérente à la répétition est encore redoublée par l'amplification inhérente à l'accroissement de la boule de neige. On peut représenter de manière identique le phénomène dans un schéma tensif. L'intensité correspond une nouvelle fois à l'émotion suscitée, à la satisfaction apportée par la répétition. Mais dans ce cas, l'étendue s'enrichit et devient forte d'un autre processus générateur d'intensité: au nombre de répétitions s'ajoute l'augmentation, ou la diminution - peu importe, l'effet est identique - de l'acteur soumis à variation (taille des personnages, nombre de personnes et place dans la piscine, par exemple pour La Piscine).

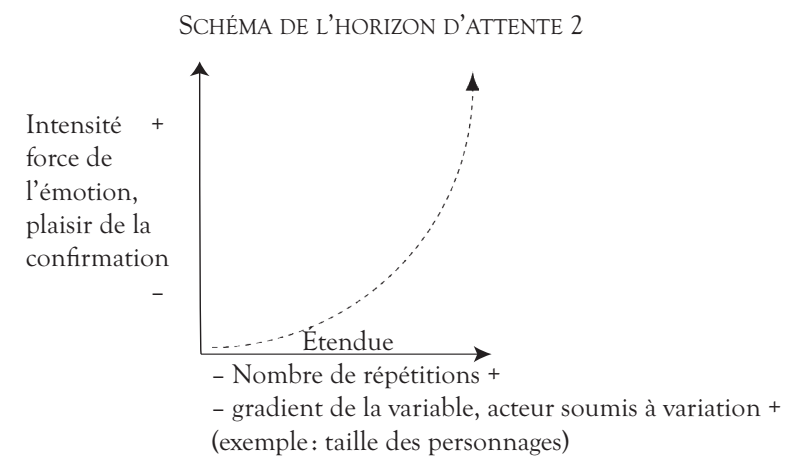

À partir de ces deux schémas, on peut risquer deux hypothèses sur le fonctionnement général de l'habitude et de la répétition dans les albums d'enfance. Tout d'abord, on peut expliquer la dimension prospective par l'orientation tensive du schéma: l'amplification n'a de sens qu'orientée du moins vers le plus, la surenchère de la répétition ne prend sens que prospectivement. Ensuite, il semble que l'on peut également expliquer la très grande fréquence du modèle de la boule de neige avec intensification par les mécanismes précédemment décrits et représentés dans des schémas tensifs. L'intensification apparaît en quelque sorte comme le prolongement le plus naturel de l'amplification déjà produite par la répétition elle-même. En la redoublant, elle la porte à son plus haut degré d'efficacité, obligeant d'ailleurs à considérer que les intensités des deux schémas se superposent, renforçant l'émotion et le plaisir de la confirmation de la répétition.

Pourquoi, enfin, ce goût de la répétition dans les albums? On peut bien sûr avancer une première explication qui découle directement des phénomènes tensifs décrits: la répétition, redoublée par l'intensification, a le mérite de générer un horizon d'attente extrêmement fort, susceptible de capter l'attention, forcément labile, des jeunes enfants. On peut aussi imaginer que le vedettariat de la répétition puise dans des fondements plus philosophiques ou psychanalytiques, que l'on ne peut qu'évoquer brièvement dans le cadre de cet article. La répétition, puissant signe de nos vies psychiques, est aussi ce qui permet de faire événement, ou de pallier aux insatisfactions de l'absence, comme dans le très célèbre jeu de la bobine analysé par Freud. Gageons que cet arrière-fond mental n'est pas absolument absent dans les albums d'enfant. La répétition dans les albums dit, peut-être, la permanence des choses, une non-évanescence rassurante du monde; elle introduit l'enfant à une profondeur événementielle qui n'est pas close sur l'instant. La fascination de la répétition est ici fascination du rituel, de l'histoire, de la permanence, de la maîtrise et du partage.

\section{LA SURPRISE COGNITIVE}

L'autre grand modèle d'album, presque opposé à celui de la répétition, puise dans les ressources de la composante dialogique. La surprise est alors non plus un effet second, né de la répétition dialectique, mais le premier objectif visé par les créateurs 
d'albums. La composante dialogique prend en charge tous les mécanismes associés traditionnellement à l'énonciation, mais Rastier y ajoute un appareil modal à grain fin, particulièrement adapté à la description des effets de surprise. La modalisation ne flotte pas sur l'ensemble du texte, mais prend corps sur un univers bien défini. De manière générale,

[...] l'univers est associé à un foyer donné (un évaluateur), qui est à la source des propositions et de leur modalisation (par exemple, un personnage ou encore plusieurs personnages, s'ils partagent exactement les mêmes croyances).

(Hébert, 2006; texte en ligne)

Plus spécifiquement, l'ensemble des effets de modalisation prennent sens par rapport à un «univers de référence». Cet univers de référence, selon Rastier, comprend

[...] les propositions vraies dans les mondes factuels de tous les univers des acteurs d'un même texte [...] associé, par défaut, à l'énonciateur représenté. (1989: 84)

L'univers modalisé devient un Monde. En fonction des modalisations, on distinguera entre monde factuel, monde contrefactuel, et monde possible.

Dans un article précédent, nous avons montré que la surprise pouvait prendre différents aspects, selon que l'observateur possède un savoir supérieur ou inférieur à celui des acteurs de l'énoncé. Nous ne prendrons qu'un exemple, celui de Tromboline et Foulbazar dans Le chat et le chien (2007), deux poussins créés par Claude Ponti, l'un des plus célèbres auteurs français d'albums d'enfance. Les albums de Tromboline et Foulbazar sont relativement courts, et édités dans un petit format facile à manipuler. Cette série d'albums, qui s'adresse à un public plus jeune que les autres productions de Ponti, joue très souvent avec les ressorts de la surprise la plus forte, construite sur un point de vue de l'observateur en position de non-savoir par rapport aux acteurs de l'énoncé. Dans l'opus choisi, l'histoire s'ouvre sur une double page qui contient en substance tous les éléments de la surprise. On découvre l'un des poussins, Tromboline, qui tient un anneau, face à un chien, qui se tient debout comme le poussin. En bas, du côté gauche de la double page, on peut lire l'énigmatique énoncé:
«Parfois Tromboline a un chien».

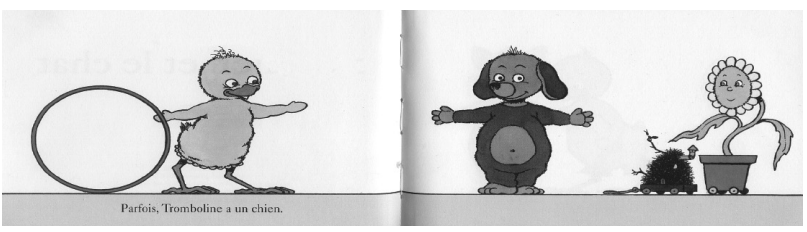

Dans cet énoncé, le lecteur averti entend une première tension entre l'aspectualisation de «avoir un chien", durative et imperfective, et l'aspectualisation du «Parfois» en début de phrase, dont les sèmes aspectuels /ponctuel/ et/itératif/sont, dans un univers adulte, difficilement compatibles avec la durativité et l'imperfectivité. Comment peut-on donc, en effet, n'avoir un chien que "parfois»? On l'a ou on ne l'a plus, mais on ne l'a pas parfois. D'emblée, le chien apparaît comme un objet magique, qui surgit dans l'univers du poussin par la seule grâce de ses désirs. Il y a dès lors coexistence de plusieurs univers qui correspondent particulièrement bien à la situation de lecture des albums. Dans l'univers de l'observateur adulte, "avoir un chien» est factuel dans l'image, mais problématique dans l'énoncé verbal. L'énoncé implique, sans long calcul, un monde contrefactuel dont l'observateur adulte peut faire l'hypothèse qu'il se rapporte à la vision de l'enfant. À l'inverse, dans l'univers enfantin, la tension aspectuelle ne fait nullement problème, car elle satisfait parfaitement aux règles de ce que Winnicott nomme un espace potentiel, ou transitionnel. Dans cet espace, que l'enfant auditeur d'albums habite encore, l'apparition de l'objet est toujours quasi magique: il survient au gré de l'enfant, dans le prolongement du sein de la mère, dont l'apparition donne à l'enfant l'illusion de toujours coïncider avec son désir. La suite de l'album va donner la clé des tensions aspectuelles et modales en conférant, à l'apparition canine, un tour moins magique, mais néanmoins sous la dépendance de la seule volonté de l'enfant poussin. Les pages suivantes, jusqu'à la cinquième double page, nous apprennent que parfois Foulbazar a un chat, que Tromboline joue avec son chien, que Foulbazar fait de même avec son chat, et que parfois le chat et le chien s'amusent ensemble. L'observateur constate que l'on n'a toujours que deux acteurs en scène. Puis, 
brutalement, «ils s'en vont», aussi magiquement qu'ils étaient apparus, en cinquième page. La dernière page donne la clé de l'ensemble: on y voit Tromboline et Foulbazar quitter leur déguisement respectif de chat et de chien. Il n'y avait bien que deux acteurs et deux déguisements, d'où l'impossibilité d'une coprésence des quatre acteurs. Le fait d'avoir un chien, ou un chat, est effectivement contrefactuel, puisqu'il relève de la mimicry, du déguisement. Le chien ou le chat ne sont pas des objets magiques, ils ne se matérialisent pas par pure pensée, mais dépendent uniquement de la décision des poussins de se déguiser: à l'illusion fait donc suite, logiquement, la désillusion, seconde face selon Winnicott de la constitution de l'espace transitionnel. En effet, constamment, l'espace transitionnel s'étend, s'ouvre au monde extérieur et à sa rationalité par l'effet de la dialectique illusion/ désillusion, et l'album des poussins semble bien faire fonds sur ce principe fondamental de l'enfance. Du point de vue discursif, on notera que la surprise oblige l'observateur à reconsidérer l'ensemble du texte qu'il vient de lire. Bien évidemment, la surprise apporte sa charge émotionnelle, mais l'essence de l'émotion est d'abord cognitive, puisque la surprise invite l'observateur à statuer rétrospectivement sur la vérité, sur l'aspect factuel ou contrefactuel de ce qui a précédé.

\section{ILLUSION DE LA RÉPÉTITION,}

\section{DÉSILLUSION DE LA SURPRISE}

Dans les albums, la répétition et la surprise sont à mi-chemin entre la généricité qui caractérise ce type de texte et les œuvres uniques créées par les auteurs. Entre les deux, ces formes typiques déclinent leurs variantes de manière presque obsédante, aucun texte ne paraissant devoir y échapper. La répétition, comme une boule de neige, offre son mouvement jubilatoire, sa dynamique prospective. Comme une comptine, elle entraîne l'enfant dans un mouvement vers l'après en jouant sur des variations plus ou moins prévisibles. Les graphes thématisés de la sémantique textuelle permettent d'enregistrer ce jeu dans toute sa complexité, tandis que les schématisations tensives représentent et expliquent la dynamique inhérente aux répétitions et leur rôle dans la constitution d'un horizon d'attente. Dans le temps de la lecture, les répétitions installent l'enfant dans l'illusion rassurante d'une histoire maîtrisée et d'un plaisir qui, par définition, ne fera pas défaut, le destin de la répétition dans l'album étant de se répéter. $\mathrm{La}$ répétition de l'album est en effet non pas une simple rime ponctuelle, une allitération plus ou moins volontaire, mais bien un moteur, un principe de construction et de progression de l'histoire. C'est une répétition de répétition, qui joue ainsi sur l'émotion du lecteur. La surprise, par un effet de symétrie saisissant, ouvre sur l'autre face du monde, l'autre vecteur de l'espace du jeu. Le coup de théâtre de la surprise est tout aussi jubilatoire que la répétition de la répétition, mais il procède, lui, rétrospectivement et fait connaitre au jeune auditeur et contemplateur des images et du texte un autre genre de joie. L'observateur enfant se découvre, dans le moment de la survenue de la surprise et de ses révélations, une compétence cognitive propre à la textualité, qui passe par la maîtrise du point de vue. La surprise rend l'enfant compétent, elle le met face au texte et lui apprend quelque chose sur ce qu'est un texte. La surprise génère, en même temps que l'étonnement, un savoir que l'on peut qualifier de métadiscursif, car il fait accéder à une forme de réflexivité par rapport à ce qu'est la totalité textuelle, à ce qui fait qu'une histoire fonctionne. La surprise est une joie d'origine cognitive, portée par une désillusion bénéfique. Dans l'univers des albums, la répétition et la surprise participent ainsi, à leur manière, avec leurs ressources propres d'illusions et de désillusions, à la construction de ce que Winnicott nomme l'espace potentiel, espace transitionnel où l'enfant se construit un monde supportable dans les premiers temps de sa vie, puis espace du jeu et enfin espace des pratiques de l'adulte. 


\section{RÉFÉRENCES BIBLIOGRAPHIQUES}

COUÉGNAS, N. [2009]: «Sémiotique tensive », dans D. Ablali et

D. Ducard (dir.), Vocabulaire des études sémiotiques et sémiologiques, Paris et Besançon, Honoré Champion et Presses universitaires de FrancheComté, 67-72;

[2010]: «L'album, un genre de texte. Contes, boules de neige, et surprise", dans A. Zinna (dir.), L'Album. Un design du sensible, à paraître dans la coll. sémiotique de l'Université Toulouse-Le Mirail.

COX, P. [2002]: Cependant... Le livre le plus court du monde, Paris, Seuil. Edy-Legrand, E. [(1919) 2000]: Macao et Cosmage, Paris, Éd.

Circonflexe.

FontanilLe, J. et C. Zilberberg [1998] : Tension et Signification, Liège, Mardaga.

HÉBERT, L. [2006]: «La dialogique onto-véridictoire», Signo. En ligne: http://www.signosemio.com/rastier/dialogique.asp\#ancreHaut (page consultée le 3 mai 2010).

HOLZWARTH, W. et W. ERLBRUCH [(1989) 1993]: De la petite taupe qui voulait savoir qui lui avait fait sur la tête, Paris, Éd. Milan.

PONTI, C. [2007]: Tromboline et Foulbazar. Le chat et le chien, Paris, L'École des loisirs.

Poussier, A. [2008] : La Piscine, Paris, L'École des loisirs.

Ramos, M. [2001]: C'est moi le plus fort, Paris, Pastel.

RASTIER, F. [1989]: Sens et Textualité, Paris, Hachette;

[2006]: «De la signification lexicale au sens textuel: éléments pour une approche unifiée», Texto!, vol.XI, no 1 . En ligne: http://www. revue-texto.net/Inedits/Rastier/Rastier_Signification-lexicale.html (page consultée le 3 mai 2010).

VAN DER Linden, S. [2006]: Lire l'album, Le Puy-en-Velay, L'atelier du poisson soluble.

WinNICOTT, D.W. [1975]: Jeu et Réalité. L'espace potentiel, Paris, Gallimard. 